\title{
Gilbert damping in noncollinear magnetic systems
}

\author{
S. Mankovsky, S. Wimmer, and H. Ebert \\ Department of Chemistry/Phys. Chemistry, LMU Munich, Butenandtstrasse 11, D-81377 Munich, Germany
}

(Received 30 May 2018; published 5 September 2018)

\begin{abstract}
The modification of the magnetization dissipation or Gilbert damping caused by an inhomogeneous magnetic structure and expressed in terms of a wave vector dependent tensor $\underline{\alpha}(\vec{q})$ is investigated by means of linear response theory. A corresponding expression for $\underline{\alpha}(\vec{q})$ in terms of the electronic Green function has been developed giving in particular the leading contributions to the Gilbert damping linear and quadratic in $q$. Numerical results for realistic systems are presented that have been obtained by implementing the scheme within the framework of the fully relativistic KKR (Korringa-Kohn-Rostoker) band structure method. Using the multilayered system $\left(\mathrm{Cu} / \mathrm{Fe}_{1-x} \mathrm{Co}_{x} / \mathrm{Pt}\right)_{n}$ as an example for systems without inversion symmetry we demonstrate the occurrence of nonvanishing linear contributions. For the alloy system bcc $\mathrm{Fe}_{1-x} \mathrm{Co}_{x}$ having inversion symmetry, on the other hand, only the quadratic contribution is nonzero. As it is shown, this quadratic contribution does not vanish even if the spin-orbit coupling is suppressed, i.e., it is a direct consequence of the noncollinear spin configuration.
\end{abstract}

DOI: 10.1103/PhysRevB.98.104406

\section{INTRODUCTION}

The magnetization dissipation in magnetic materials is conventionally characterized by means of the Gilbert damping (GD) tensor $\underline{\alpha}$ that enters the Landau-Lifshitz-Gilbert (LLG) equation [1]. This positive-definite second-rank tensor depends in general on the magnetization direction. It is well established that in the case of spatially uniformly magnetized ferromagnetic (FM) metals two regimes of slow magnetization dynamics can be distinguished, which are governed by different mechanisms of dissipation [2-4]: a conductivitylike behavior occurring in the limiting case of ordered compounds that may be connected to the Fermi breathing mechanism and a resistivitylike behavior shown by materials with appreciable structural, chemical, or temperature induced disorder and connected to a spin-flip scattering mechanism. An important issue is that both mechanisms are determined by the spin-orbit coupling in the system (see, e.g., Refs. [2,4,5]). During the last years, it was demonstrated by various authors that firstprinciples calculations of the GD parameter for collinear ferromagnetic materials allow us to cover both regimes without use of any phenomenological parameters. In fact, in spite of the differences concerning the formulation for the damping parameter and the corresponding implementation [6-8], the numerical results are in general in rather good agreement with each other as well as with experiment.

In the case of a pronounced noncollinear magnetic texture, e.g., in the case of domain walls or topologically nontrivial magnetic configurations like skyrmions, the description of the magnetization dissipation assuming a spatial-invariant tensor $\alpha$ is incomplete, and a nonlocal character of GD tensor in such systems has to be taken into account [9-11]. This implies that the dissipative torque on the magnetization should be represented by the expression of the following general form [12]:

$$
\tau_{G D}=\hat{m}(\vec{r}, t) \times \int d^{3} r^{\prime} \underline{\alpha}\left(\vec{r}-\vec{r}^{\prime}\right) \frac{\partial}{\partial t} \hat{m}\left(\vec{r}^{\prime}, t\right) .
$$

In the case of a magnetic texture varying slowly in space, however, an expansion of the damping parameter in terms of the magnetization density and its gradients [11] is nevertheless appropriate:

$$
\begin{aligned}
\alpha_{i j}= & \alpha_{i j}+\alpha_{i j}^{k l} m_{k} m_{l}+\alpha_{i j}^{k l p} m_{k} \frac{\partial}{\partial r_{l}} m_{p} \\
& +\alpha_{i j}^{k l p q} \frac{\partial}{\partial r_{k}} m_{l} \frac{\partial}{\partial r_{p}} m_{q}+\cdots,
\end{aligned}
$$

where the first term $\alpha_{i j}$ stands for the conventional isotropic GD and the second term $\alpha_{i j}^{k l} m_{k} m_{l}$ is associated with the magnetocrystalline anisotropy (MCA). The third so-called chiral term $\alpha_{i j}^{k l p} m_{k} \frac{\partial}{\partial r_{l}} m_{p}$ is nonvanishing in noncentrosymmetric systems. The important role of this contribution was discussed theoretically [13] as well as demonstrated experimentally when investigating the field-driven domain wall (DW) motion in asymmetric $\mathrm{Pt} / \mathrm{Co} / \mathrm{Pt}$ trilayers [14].

As an alternative to the expansion in Eq. (2) one can discuss the Fourier transform $\alpha(\vec{q})$ of the damping parameter characterizing inhomogeneous magnetic systems, which enters the expression for transverse magnetization dynamics induced by a transverse external perturbation field $h(\vec{r}, t)$. This is derived via the linearized LLG equation [15]

$$
\vec{m}(\vec{q}, \omega)=\frac{\gamma h(\vec{q}, \omega)}{\omega_{0}(\vec{q})-\omega-i \alpha(\vec{q}) \omega},
$$

where $\omega_{0}(\vec{r}) \hat{z}=\gamma \vec{H}_{\text {eff }}(\vec{r})$, and an effective field $\vec{H}_{\text {eff }}(\vec{r})$ is contributed by an external magnetic field, as well as effective fields associated with the exchange interactions and magnetic anisotropy [15]. In this formulation the term linear in $q$ is the first chiral term appearing in the expansion of $\underline{\alpha}(\vec{q})$ in powers of $q$. Furthermore, it is important to note that it is directly connected to the $\alpha_{i j}^{k l p} m_{k} \frac{\partial}{\partial r_{l}} m_{p}$ term in Eq. (2).

By applying a gauge field theory, the origin of the noncollinear corrections to the GD can be ascribed to the emergent electromagnetic field created in the time-dependent mag- 
netic texture [16,17]. Such an emergent electromagnetic field gives rise to a spin current whose divergence characterizes the change of the angular momentum in the system. This allows us to discuss the impact of noncollinearity on the GD via a spin-pumping formulation $[9,16,18]$. Some details of the physics behind this effect depend on the specific properties of the material considered. Accordingly, different models for magnetization dissipation were discussed in the literature [9,12,16,19-21]. Noncentrosymmetric two-dimensional systems for which the Rashba-like spin-orbit coupling plays an important role have received special interest in this context. They have been discussed in particular by Akosa et al. [21], in order to explain the origin of chiral GD in the presence of a chiral magnetic structure.

The fourth term on the r.h.s. of Eq. (2) corresponds to a quadratic term of an expansion of $\underline{\alpha}(\vec{q})$ with respect to $q$. It was investigated for bulk systems with nonmagnetic [22] and magnetic [9] impurity atoms, for which the authors have shown on the basis of model consideration that it can give a significant correction to the homogeneous GD in the case of weak metallic ferromagnets. In striking contrast to the uniform part of the GD this contribution does not require a nonvanishing spin-orbit interaction.

To our knowledge, only very few ab initio investigations on the Gilbert damping in noncollinear magnetic systems along the lines sketched above have been reported so far in the literature. Yuan et al. [23] calculated the in-plane and out-ofplane damping parameters in terms of the scattering matrix for permalloy in the presence of Néel and Bloch domain walls. Freimuth et al. [24] discuss the properties of a $q$-dependent Gilbert damping $\underline{\alpha}(\vec{q})$ calculated for the one-dimensional Rashba model in the presence of the Néel-type noncollinear magnetic exchange field, demonstrating different GD for lefthanded and right-handed DWs. Here we extend the formalism developed before to deal with the GD in ferromagnets [6], to get access to the noncollinear system. The formalism based on linear response theory allows us to expand the GD parameters with respect to a modulation of the magnetization expressed in terms of a wave vector $\vec{q}$. Corresponding numerical results will be presented and discussed.

\section{GILBERT DAMPING FOR NONCOLLINEAR MAGNETIZATION}

In the following we focus on the intrinsic contribution to the Gilbert damping, excluding spin current induced magnetization dissipation which occurs in the presence of an external electric field. For the considerations on the magnetization dissipation an adiabatic variation of the magnetization in the time and space domain is assumed. Moreover, it is assumed that the magnitude of the local magnetic moments is unchanged during a change of the magnetization, i.e., the exchange field should be strong enough to separate transverse and longitudinal parts of the magnetic susceptibility. With these restrictions, the nonlocal Gilbert damping can be determined in terms of the spin susceptibility tensor

$$
\chi_{\alpha \beta}(\vec{q}, \omega)=i \frac{1}{V} \int_{0}^{\infty} d t\left\langle\hat{S}_{\alpha}(\vec{q}, t) \hat{S}_{\beta}(-\vec{q}, 0)\right\rangle_{0} e^{i(\omega-\delta) t},
$$

where $\hat{S}_{\alpha}(\vec{q}, t)$ is the $\vec{q}$ - and $t$-dependent spin operator and reduced units have been used $(\hbar=1)$.

With this, the Fourier transformation of the real-space Gilbert damping can be represented by the expression [25,26]

$$
\alpha_{\alpha \beta}(\vec{q})=\frac{\gamma}{M_{0} V} \lim _{\omega \rightarrow 0} \frac{\partial \Im\left[\chi^{-1}\right]_{\alpha \beta}(\vec{q}, \omega)}{\partial \omega} .
$$

Here $\gamma=g \mu_{B}$ is the gyromagnetic ratio, $M_{0}=\mu_{\text {tot }} \mu_{B} / V$ is the equilibrium magnetization, and $V$ is the volume of the system. In order to avoid the calculation of the dynamical magnetic susceptibility tensor $\chi(\vec{q}, \omega)$, which is the Fourier transformed of the real space susceptibility $\chi\left(\vec{r}-\vec{r}^{\prime}, \omega\right)$, it is convenient to represent $\chi(\vec{q}, \omega)$ in Eq. (5), in terms of a correlation function of time derivatives of $\hat{S}$. As $\dot{\hat{S}}$ corresponds to the torque $\overrightarrow{\mathcal{T}}$, that may include nondissipative and dissipative parts, one may consider instead the torque-torque correlation function $\underline{\pi}(\vec{q}, \omega)[26-29]$.

Assuming the magnetization direction parallel to $\hat{z}$ one obtains the expression for the Gilbert damping $\underline{\alpha}(\vec{q})$

$$
\underline{\alpha}(\vec{q})=\frac{\gamma}{M_{0} V} \lim _{\omega \rightarrow 0} \frac{\partial \Im[\underline{\epsilon} \cdot \underline{\pi}(\vec{q}, \omega) \cdot \underline{\epsilon}]}{\partial \omega},
$$

where $\underline{\epsilon}=\left[\begin{array}{cc}0 & 1 \\ -1 & 0\end{array}\right]$ is the transverse Levi-Civita tensor. This implies the following relationship of the $\underline{\alpha}$ tensor elements with the elements of the torque-torque correlation tensor $\pi$ : $\alpha_{x x} \sim-\pi_{y y}$ and $\alpha_{y y} \sim-\pi_{x x}$ [26].

Using Kubo's linear response theory in the Matsubara representation and taking into account the translational symmetry of a solid the torque-torque correlation function $\pi_{\alpha \beta}(\vec{q}, \omega)$ can be expressed by (see, e.g., Ref. [30]):

$$
\pi_{\alpha \beta}\left(\vec{q}, i \omega_{n}\right)=\frac{1}{\beta} \sum_{p_{m}}\left\langle\mathcal{T}^{\alpha} \mathcal{G}\left(\vec{k}+\vec{q}, i \omega_{n}+i p_{m}\right) \mathcal{T}^{\beta} \mathcal{G}\left(\vec{k}, i p_{m}\right)\right\rangle_{c},
$$

where $\mathcal{G}(\vec{k}, i p)$ is the Matsubara Green function and $\langle\ldots\rangle_{c}$ indicates a configurational average required in the presence of any disorder (chemical, structural, or magnetic) in the system. Using a Lehman representation for the Green function [30]

$$
\mathcal{G}\left(\vec{k}, i p_{m}\right)=\int_{-\infty}^{+\infty} \frac{d E}{\pi} \frac{\Im G^{+}(\vec{k}, E)}{i p_{m}-E}
$$

with $G^{+}(\vec{k}, E)$ the retarded Green function and using the relation

$$
\frac{1}{\beta} \sum_{p_{m}} \frac{1}{i p_{m}+i \omega_{n}-E_{1}} \frac{1}{i p_{m}-E_{2}}=\frac{f\left(E_{2}\right)-f\left(E_{1}\right)}{i \omega_{n}+E_{2}-E_{1}}
$$

for the sum over the Matsubara poles in Eq. (7), the torquetorque correlation function is obtained as:

$$
\begin{aligned}
\pi_{\alpha \beta}\left(\vec{q}, i \omega_{n}\right)= & \frac{1}{\Omega_{B Z}} \int d^{3} k \int_{-\infty}^{+\infty} \frac{d E_{1}}{\pi} \\
& \times \int_{-\infty}^{+\infty} \frac{d E_{2}}{\pi} \operatorname{Tr}\left\langle\mathcal{T}^{\alpha} \Im G\left(\vec{k}, E_{1}\right) \mathcal{T}^{\beta} \Im G\left(\vec{k}, E_{2}\right)\right. \\
& \left.\times \frac{f\left(E_{2}\right)-f\left(E_{1}\right)}{i \omega_{n}+E_{2}-E_{1}}\right\rangle_{c}
\end{aligned}
$$


Performing finally the analytical continuation $i \omega_{n} \rightarrow \omega+i \delta$ one arrives at the expression

$$
\begin{aligned}
\Gamma_{\alpha \beta}(\vec{q}, \omega) & =-\frac{\pi}{\Omega_{B Z}} \int d^{3} k \int_{-\infty}^{+\infty} \frac{d E_{1}}{\pi} \int_{-\infty}^{+\infty} \frac{d E_{2}}{\pi} \operatorname{Tr}\left\langle\mathcal{T}^{\alpha} \Im G\left(\vec{k}+\vec{q}, E_{1}\right) \mathcal{T}^{\beta} \Im G\left(\vec{k}, E_{2}\right)\right\rangle_{c}\left(f\left(E_{2}\right)-f\left(E_{1}\right)\right) \delta\left(\omega+E_{2}-E_{1}\right) \\
& =-\frac{\pi}{\Omega_{B Z}} \int d^{3} k \int_{-\infty}^{+\infty} \frac{d E}{\pi} \operatorname{Tr}\left\langle\mathcal{T}^{\alpha} \Im G(\vec{k}+\vec{q}, E) \mathcal{T}^{\beta} \Im G(\vec{k}, E+\omega)\right\rangle_{c}(f(E)-f(E+\omega))
\end{aligned}
$$

for the imaginary part of the correlation function with $\Gamma_{\alpha \beta}(\vec{q}, \omega)=-\pi \Im \pi_{\alpha \beta}(\vec{q}, \omega)$. Accordingly one gets for the diagonal elements of Gilbert damping tensor the expression

$$
\begin{aligned}
\alpha_{\alpha \alpha}(\vec{q}) & =\left.\frac{\gamma}{M_{0} V} \lim _{\omega \rightarrow 0} \frac{\partial[\underline{\epsilon} \cdot \underline{\Gamma}(\vec{q}, \omega) \cdot \underline{\epsilon}]}{\partial \omega}\right|_{\alpha \alpha} \\
& =\frac{\gamma \pi}{M_{0} V} \lim _{\omega \rightarrow 0} \frac{\partial}{\partial \omega} \frac{1}{\Omega_{B Z}} \int d^{3} k \int_{-\infty}^{+\infty} \frac{d E}{\pi^{2}}(f(E+\omega)-f(E)) \operatorname{Tr}\left\langle\mathcal{T}^{\beta} \Im G(\vec{k}+\vec{q}, E) \mathcal{T}^{\beta} \Im G(\vec{k}, E+\omega)\right\rangle_{c} \\
& =\frac{\gamma}{M_{0} V} \frac{1}{\Omega_{B Z}} \int d^{3} k \int_{-\infty}^{+\infty} \frac{d E}{\pi} \delta\left(E-E_{F}\right) \operatorname{Tr}\left\langle\mathcal{T}^{\beta} \Im G(\vec{k}+\vec{q}, E) \mathcal{T}^{\beta} \Im G(\vec{k}, E)\right\rangle_{c} \\
& =\frac{1}{4}\left[\alpha_{\alpha \alpha}\left(\vec{q}, G^{+}, G^{+}\right)+\alpha_{\alpha \alpha}\left(\vec{q}, G^{-}, G^{-}\right)-\alpha_{\alpha \alpha}\left(\vec{q}, G^{+}, G^{-}\right)-\alpha_{\alpha \alpha}\left(\vec{q}, G^{-}, G^{+}\right)\right],
\end{aligned}
$$

where the index $\beta$ of the torque operator $\mathcal{T}^{\beta}$ is related to the index $\alpha$ according to Eq. (6), and the auxiliary functions

$$
\alpha_{\alpha \alpha}\left(\vec{q}, G^{ \pm}, G^{ \pm}\right)=\frac{\gamma}{M_{0} V \pi} \frac{1}{\Omega_{B Z}} \int d^{3} k \operatorname{Tr}\left\langle\mathcal{T}^{\beta} G^{ \pm}\left(\vec{k}+\vec{q}, E_{F}\right) \mathcal{T}^{\beta} G^{ \pm}\left(\vec{k}, E_{F}\right)\right\rangle_{c}
$$

expressed in terms of the retarded and advanced Green functions, $G^{+}$and $G^{-}$, respectively.

To account properly for the impact of spin-orbit coupling when dealing with Eqs. (11) and (12) a description of the electronic structure based on the fully relativistic Dirac formalism is used. Working within the framework of local spin density formalism (LSDA) this implies for the Hamiltonian the form [31]:

$$
\hat{H}_{D}=c \overrightarrow{\boldsymbol{\alpha}} \cdot \vec{p}+\beta m c^{2}+V(\vec{r})+\beta \overrightarrow{\boldsymbol{\sigma}} \cdot \hat{\vec{m}} B_{x c}(\vec{r}) .
$$

Here $\alpha_{i}$ and $\beta$ are the standard Dirac matrices, $\vec{\sigma}$ denotes the vector of relativistic Pauli matrices, $\vec{p}$ is the relativistic momentum operator [32], and the functions $V(\vec{r})$ and $\vec{B}_{x c}=\vec{\sigma} \cdot \overrightarrow{\hat{m}} B_{x c}(\vec{r})$ are the spin-averaged and spin-dependent parts, respectively, of the LSDA potential [33] with $\hat{\vec{m}}$ giving the orientation of the magnetization.

With the Dirac Hamiltonian given by Eq. (13), the torque operator may be written as $\overrightarrow{\mathcal{T}}=\beta[\vec{\sigma} \times \vec{m}] B_{x c}(\vec{r})$. Furthermore, the Green functions entering Eqs. (11) and (12) are determined using the spin-polarized relativistic version of multiple scattering theory $[31,34]$ with the real space representation of the retarded Green function given by:

$$
\begin{aligned}
G^{+}\left(\vec{r}, \vec{r}^{\prime}, E\right)= & \sum_{\Lambda \Lambda^{\prime}} Z_{\Lambda}^{n}(\vec{r}, E) \tau_{\Lambda \Lambda^{\prime}}^{n m}(E) Z_{\Lambda^{\prime}}^{m \times}\left(\vec{r}^{\prime}, E\right) \\
& -\delta_{n m} \sum_{\Lambda}\left[Z_{\Lambda}^{n}(\vec{r}, E) J_{\Lambda^{\prime}}^{n \times}\left(\vec{r}^{\prime}, E\right) \Theta\left(r_{n}^{\prime}-r_{n}\right)+J_{\Lambda}^{n}(\vec{r}, E) Z_{\Lambda^{\prime}}^{n \times}\left(\vec{r}^{\prime}, E\right) \Theta\left(r_{n}-r_{n}^{\prime}\right)\right] .
\end{aligned}
$$

Here $\vec{r}, \vec{r}^{\prime}$ refer to atomic cells centered at sites $n$ and $m$, respectively, where $Z_{\Lambda}^{n}(\vec{r}, E)=Z_{\Lambda}\left(\vec{r}_{n}, E\right)=Z_{\Lambda}\left(\vec{r}-\vec{R}_{n}, E\right)$ is a function centered at the corresponding lattice vector $\vec{R}_{n}$. The four-component wave functions $Z_{\Lambda}^{n}(\vec{r}, E)\left(J_{\Lambda}^{n}(\vec{r}, E)\right)$ are regular (irregular) solutions to the single-site Dirac equation labeled by the combined quantum numbers $\Lambda=(\kappa, \mu)$, with $\kappa$ and $\mu$ being the spin-orbit and magnetic quantum numbers [32]. Finally, $\tau_{\Lambda \Lambda^{\prime}}^{n m}(E)$ is the so-called scattering path operator that transfers an electronic wave coming in at site $m$ into a wave going out from site $n$ with all possible intermediate scattering events accounted for.

Using matrix notation with respect to $\Lambda$, this leads to the following expression for the auxiliary damping parameters in Eq. (12):

$$
\alpha_{\alpha \alpha}\left(\vec{q}, G^{ \pm}, G^{ \pm}\right)=\frac{\gamma}{M_{0} V \pi} \frac{1}{\Omega_{B Z}} \int d^{3} k \operatorname{Tr}\left\langle\underline{T}^{\beta} \underline{\tau}\left(\vec{k}+\vec{q}, E_{F}^{ \pm}\right) \underline{T}^{\beta} \underline{\tau}\left(\vec{k}, E_{F}^{ \pm}\right)\right\rangle_{c} .
$$

In the case of a uniform magnetization, i.e., for $q=0$ one obviously gets an expression for the Gilbert damping tensor as it was worked out before [7]. Assuming small wave vectors, the term $\underline{\tau}\left(\vec{k}+\vec{q}, E_{F}^{ \pm}\right)$can be expanded w.r.t. $\vec{q}$ leading to the series

$$
\underline{\tau}\left(\vec{k}+\vec{q}, E_{F}\right)=\underline{\tau}(\vec{k}, E)+\sum_{\mu} \frac{\partial \underline{\tau}(\vec{k}, E)}{\partial k_{\mu}} q_{\mu}+\frac{1}{2} \sum_{\mu \nu} \frac{\partial \underline{\tau}(\vec{k}, E)}{\partial k_{\mu} \partial k_{\nu}} q_{\mu} q_{\nu}+\cdots
$$


that results in a corresponding expansion for the Gilbert damping:

$$
\underline{\alpha}(\vec{q})=\underline{\alpha}+\sum_{\mu} \underline{\alpha}^{\mu} q_{\mu}+\frac{1}{2} \sum_{\mu \nu} \underline{\alpha}^{\mu v} q_{\mu} q_{\nu}+\cdots
$$

with the following expansion coefficients:

$$
\begin{gathered}
\alpha_{\alpha \alpha}^{0 \pm \pm}=\frac{g}{\pi \mu_{\mathrm{tot}}} \frac{1}{\Omega_{B Z}} \operatorname{Trace} \int d^{3} k\left\langle\underline{T}_{\beta} \underline{\tau}\left(\vec{k}, E_{F}^{ \pm}\right) \underline{T} \beta \underline{\tau}\left(\vec{k}, E_{F}^{ \pm}\right)\right\rangle_{c} \\
\alpha_{\alpha \alpha}^{\mu \pm \pm}=\frac{g}{\pi \mu_{\mathrm{tot}}} \frac{1}{\Omega_{B Z}} \operatorname{Trace} \int d^{3} k\left\langle\underline{T}^{\beta} \frac{\partial \underline{\tau}\left(\vec{k}, E_{F}^{ \pm}\right)}{\partial k_{\mu}} \underline{T}^{\beta} \underline{\tau}\left(\vec{k}, E_{F}^{ \pm}\right)\right\rangle_{c} \\
\alpha_{\alpha \alpha}^{\mu \nu \pm \pm}=\frac{g}{\pi \mu_{\mathrm{tot}}} \frac{1}{2 \Omega_{B Z}} \operatorname{Trace} \int d^{3} k\left\langle\underline{T}^{\beta} \frac{\partial^{2} \underline{\tau}\left(\vec{k}, E_{F}^{ \pm}\right)}{\partial k_{\mu} \partial k_{v}} \underline{T}^{\beta} \underline{\tau}\left(\vec{k}, E_{F}^{ \pm}\right)\right\rangle_{c},
\end{gathered}
$$

and with the $g$ factor $2\left(1+\mu_{\mathrm{orb}} / \mu_{\text {spin }}\right)$ in terms of the spin and orbital moments, $\mu_{\text {spin }}$ and $\mu_{\mathrm{orb}}$, respectively, and the total magnetic moment $\mu_{\mathrm{tot}}=\mu_{\mathrm{spin}}+\mu_{\mathrm{orb}}$. The numerically cumbersome term in Eq. (20), that involves the second order derivative of the matrix of $\vec{k}$-dependent scattering path operator $\tau(\vec{k}, E)$, can be reformulated by means of an integration by parts:

$$
\begin{aligned}
& \frac{1}{\Omega_{B Z}} \int d^{3} k \underline{T}_{\beta} \underline{\tau}\left(\vec{k}, E_{F}\right) \underline{T}_{\beta} \frac{\partial^{2} \underline{\tau}\left(\vec{k}, E_{F}\right)}{\partial k_{\mu} \partial k_{v}}
\end{aligned}
$$

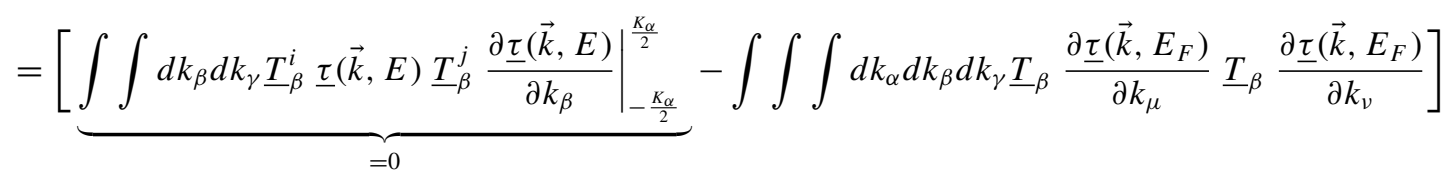

$$
\begin{aligned}
& =-\frac{1}{\Omega_{B Z}} \int d^{3} k \underline{T}_{\beta} \frac{\partial \underline{\tau}\left(\vec{k}, E_{F}\right)}{\partial k_{\mu}} \underline{T}_{\beta} \frac{\partial \underline{\tau}\left(\vec{k}, E_{F}\right)}{\partial k_{v}}
\end{aligned}
$$

leading to the much more convenient expression:

$$
\alpha_{\alpha \alpha}^{\mu \nu \pm \pm}=-\frac{g}{2 \pi \mu_{\mathrm{tot}}} \int d^{3} k \operatorname{Tr}\left\langle\underline{T^{\beta}} \frac{\partial \underline{\tau}\left(\vec{k}, E_{F}^{ \pm}\right)}{\partial k_{\mu}} \underline{T}^{\beta} \frac{\partial \underline{\tau}\left(\vec{k}, E_{F}^{ \pm}\right)}{\partial k_{\nu}}\right\rangle_{c} .
$$

\section{RESULTS AND DISCUSSIONS}

The scheme presented above to deal with the Gilbert damping in noncollinear systems has been implemented within the SPR-KKR program package [35]. To examine the importance of the chiral correction to the Gilbert damping a first application of Eq. (19) has been made for the multilayer system $\left(\mathrm{Cu} / \mathrm{Fe}_{1-x} \mathrm{Co}_{x} / \mathrm{Pt}\right)_{n}$ seen as a noncentrosymmetric model system. The calculated zero-order (uniform) GD parameter $\alpha_{x x}$ and the corresponding first-order (chiral) $\alpha_{x x}^{x}$ correction term for $\vec{q} \| \hat{x}$ are plotted in Fig. 1 top and bottom, respectively, as a function of the Co concentration $x$.

Both terms, $\alpha_{x x}$ and $\alpha_{x x}^{x}$, increase approaching the pure limits w.r.t. the $\mathrm{Fe}_{1-x} \mathrm{Co}_{x}$ alloy subsystem. In the case of the uniform parameter $\alpha_{x x}$, this increase is associated with the dominating breathing Fermi-surface damping mechanism. This implies that the modification of the Fermi surface (FS) induced by the spin-orbit coupling (SOC) follows the magnetization direction that slowly varies with time. An additional contribution to the GD, having a similar origin, occurs for the noncentrosymmertic systems with helimagnetic structure. In this case, the features of the electronic structure governed by the lack of inversion symmetry result in a FS modification dependent on the helicity of the magnetic structure. This implies a chiral contribution to the GD which can be associated with the term proportional to the gradient of
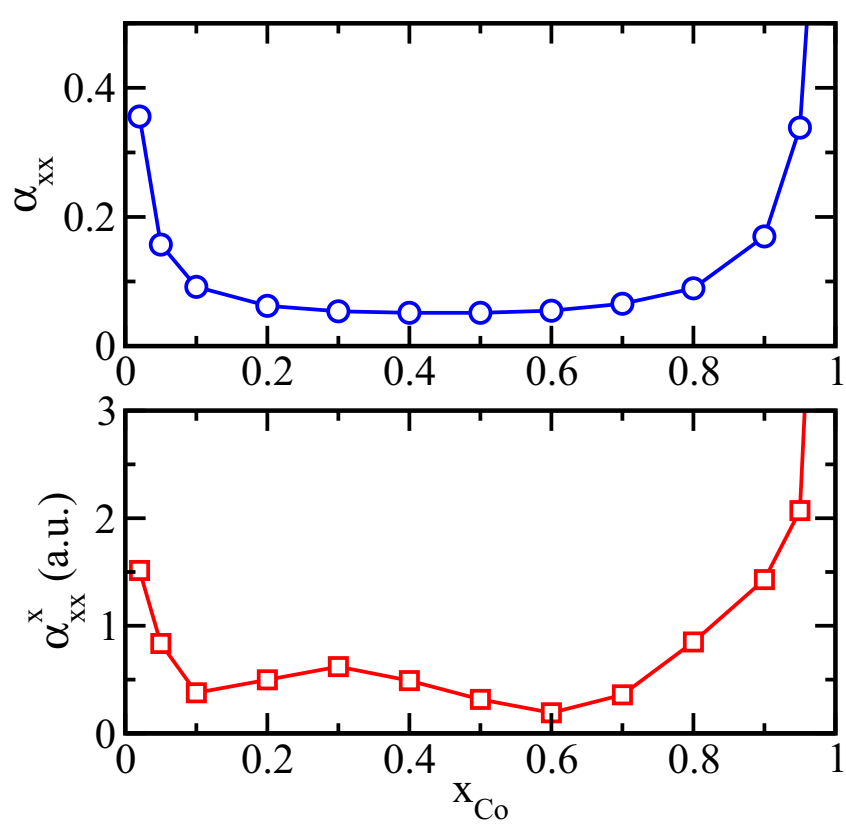

FIG. 1. The Gilbert damping parameters $\alpha_{x x}$ (top) and $\alpha_{x x}^{x}$ (bottom) calculated for the model multilayer system $\left(\mathrm{Cu} / \mathrm{Fe}_{1-x} \mathrm{Co}_{x} / \mathrm{Pt}\right)_{n}$ using Eqs. (18) and (19), respectively. 


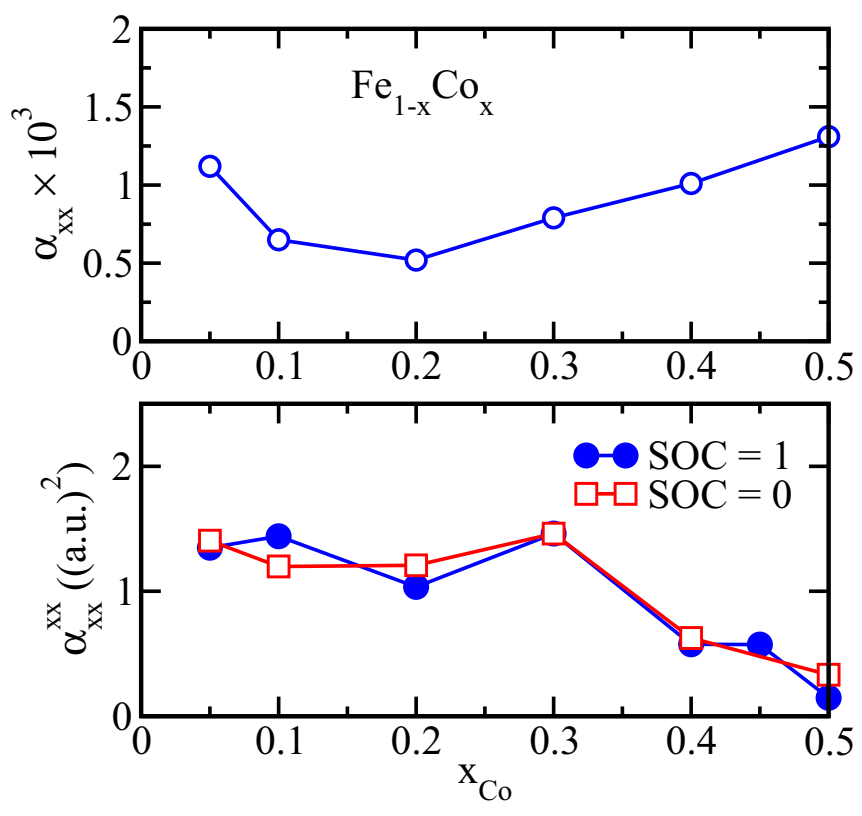

FIG. 2. The Gilbert damping terms $\alpha_{x x}$ (top) and $\alpha_{x x}^{x x}$ (bottom) calculated for bcc $\mathrm{Fe}_{1-x} \mathrm{Co}_{x}$.

the magnetization. Obviously, this additional modification of the FS and the associated mechanism for the GD does not show up for a uniform ferromagnet. As $\underline{\alpha}$ is caused by the SOC one can expect that it vanishes for vanishing SOC. This was indeed demonstrated before [5]. The same holds also for $\underline{\alpha}^{x}$ that is caused by SOC as well.

It is worth noting here that in general the term linear in $\vec{q}, \underline{\alpha}^{x} \vec{q}$, can be negative and larger by magnitude than other two positive contributions to the Gilbert damping, leading in this case to a negative total Gilbert damping. However, an expansion of GD in powers of $\vec{q}$ vector with the coefficients given by Eqs. (18)-(20) implies that the $q$ values should be small enough to ensure a positive value of the total Gilbert damping. In other words, for the regime with a magnitude of $q$ vector leading to nonphysical negative total GD the present approach is not suited anymore.

Another system considered is the ferromagnetic alloy system bcc $\mathrm{Fe}_{1-x} \mathrm{Co}_{x}$. As this system has inversion symmetry the first-order term $\underline{\alpha}^{\mu}$ should vanish. This expectation could also be confirmed by calculations that account for the SOC. The next nonvanishing term of the expansion of the GD is the term $\propto q^{2}$. The corresponding second-order term $\alpha_{x x}^{x x}$ is plotted in Fig. 2 (bottom) together with the zero-order term $\alpha_{x x}$ (top).

The bottom panel shows in addition results for $\alpha_{x x}^{x x}$ that have been obtained by calculations with the SOC suppressed. As one notes the results for the full SOC and for SOC suppressed are very close to each other. The small difference between the curves for that reason have to be ascribed to the hybridization of the spin-up and spin-down subsystems due to SOC. As discussed in the literature $[9,19,22]$ a noncollinear magnetic texture has a corresponding consequence but a much stronger impact here. In contrast to the GD in uniform FM systems where SOC is required to break the total spin conservation in the system, $\alpha_{x x}^{x x}$ is associated with the spin-pumping effect that can be ascribed to an emergent electric field created in the nonuniform magnetic system. In this case magnetic dissipation occurs due to the misalignment of the electron spin following the dynamic magnetic profile and the magnetization orientation at each atomic site, leading to the dephasing of electron spins [18].

\section{SUMMARY}

To summarize, expressions for corrections to the GD of homogeneous systems were derived which are expected to contribute in the case of noncollinear magnetic systems. The expression for the GD parameter $\alpha(\vec{q})$ seen as a function of the wave vector $\vec{q}$ is expanded in powers of $q$. In the limit of weakly varying magnetic textures, this leads to the standard uniform term, $\underline{\alpha}$, and the first- and second-order corrections, $\underline{\alpha}^{\mu}$ and $\underline{\alpha}^{\mu \nu}$, respectively. Model calculations confirmed that a nonvanishing value for $\underline{\alpha}^{\mu}$ can be expected for systems without inversion symmetry. In addition, SOC has been identified as the major source for this term. The second-order term, on the other hand, may also show up for systems with inversion symmetry. In this case it was demonstrated by numerical work that SOC plays only a minor role for $\underline{\alpha}^{\mu \nu}$, while the noncollinearity of the magnetization plays the central role.

\section{ACKNOWLEDGMENT}

Financial support by the DFG via SFB 1277 (Emergente relativistische Effekte in der Kondensierten Materie) is gratefully acknowledged.
[1] T. L. Gilbert, IEEE Trans. Magn. 40, 3443 (2004).

[2] V. Kambersky, Can. J. Phys. 48, 2906 (1970).

[3] M. Fähnle and D. Steiauf, Phys. Rev. B 73, 184427 (2006).

[4] K. Gilmore, Y. U. Idzerda, and M. D. Stiles, Phys. Rev. Lett. 99, 027204 (2007).

[5] S. Mankovsky, D. Ködderitzsch, G. Woltersdorf, and H. Ebert, Phys. Rev. B 87, 014430 (2013).

[6] A. A. Starikov, P. J. Kelly, A. Brataas, Y. Tserkovnyak, and G. E. W. Bauer, Phys. Rev. Lett. 105, 236601 (2010).

[7] H. Ebert, S. Mankovsky, D. Ködderitzsch, and P. J. Kelly, Phys. Rev. Lett. 107, 066603 (2011).
[8] I. Turek, J. Kudrnovský, and V. Drchal, Phys. Rev. B 92, 214407 (2015).

[9] Y. Tserkovnyak, E. M. Hankiewicz, and G. Vignale, Phys. Rev. B 79, 094415 (2009).

[10] K. M. D. Hals, K. Flensberg, and M. S. Rudner, Phys. Rev. B 92, 094403 (2015).

[11] K. M. D. Hals and A. Brataas, Phys. Rev. B 89, 064426 (2014).

[12] N. Umetsu, D. Miura, and A. Sakuma, Phys. Rev. B 91, 174440 (2015).

[13] K.-W. Kim, H.-W. Lee, K.-J. Lee, K. Everschor-Sitte, O. Gomonay, and J. Sinova, Phys. Rev. B 97, 100402 (2018). 
[14] E. Jue, C. K. Safeer, M. Drouard, A. Lopez, P. Balint, L. BudaPrejbeanu, O. Boulle, S. Auffret, A. Schuhl, A. Manchon et al., Nat. Mater. 15, 272 (2015).

[15] H. J. Skadsem, Y. Tserkovnyak, A. Brataas, and G. E. W. Bauer, Phys. Rev. B 75, 094416 (2007).

[16] S. Zhang and S. S.-L. Zhang, Phys. Rev. Lett. 102, 086601 (2009).

[17] G. Tatara and N. Nakabayashi, J. Appl. Phys. 115, 172609 (2014).

[18] Y. Tserkovnyak and M. Mecklenburg, Phys. Rev. B 77, 134407 (2008).

[19] J. Zang, M. Mostovoy, J. H. Han, and N. Nagaosa, Phys. Rev. Lett. 107, 136804 (2011).

[20] T. Chiba, G. E. W. Bauer, and S. Takahashi, Phys. Rev. B 92, 054407 (2015).

[21] C. A. Akosa, I. M. Miron, G. Gaudin, and A. Manchon, Phys. Rev. B 93, 214429 (2016).

[22] E. M. Hankiewicz, G. Vignale, and Y. Tserkovnyak, Phys. Rev. B 78, 020404 (2008).

[23] Z. Yuan, K. M. D. Hals, Y. Liu, A. A. Starikov, A. Brataas, and P. J. Kelly, Phys. Rev. Lett. 113, 266603 (2014).
[24] F. Freimuth, S. Blügel, and Y. Mokrousov, Phys. Rev. B 96, 104418 (2017).

[25] Z. Qian and G. Vignale, Phys. Rev. Lett. 88, 056404 (2002).

[26] E. M. Hankiewicz, G. Vignale, and Y. Tserkovnyak, Phys. Rev. B 75, 174434 (2007).

[27] H. Mori and K. Kawasaki, Prog. Theor. Phys. 27, 529 (1962).

[28] G. Mahan, J. Phys. Chem. Solids 31, 1477 (1970).

[29] M. Hasegawa, J. Phys. Soc. Jpn. 31, 649 (1971).

[30] G. D. Mahan, Many-particle physics, Physics of Solids and Liquids (Springer, New York, 2000).

[31] H. Ebert, in Electronic Structure and Physical Properties of Solids, edited by H. Dreyssé (Springer, Berlin, 2000), Vol. 535 of Lecture Notes in Physics, p. 191.

[32] M. E. Rose, Relativistic Electron Theory (Wiley, New York, 1961).

[33] A. H. MacDonald and S. H. Vosko, J. Phys. C 12, 2977 (1979).

[34] H. Ebert, J. Braun, D. Ködderitzsch, and S. Mankovsky, Phys. Rev. B 93, 075145 (2016).

[35] H. Ebert et al., The Munich SPR-KKR package, version 7.7, http://olymp.cup.uni-muenchen.de/ak/ebert/SPRKKR (2017). 